“ (C) 2012 IEEE. Personal use of this material is permitted. Permission from IEEE must be obtained for all other uses, in any current or future media, including

reprinting/republishing this material for advertising or promotional purposes, creating new collective works, for resale or redistribution to servers or lists, or reuse of any copyrighted component of this work in other works." 


\section{SPICE Steady State Modelling of Thermoelectric Generators involving the Thomson Effect}

\author{
Ian Laird \\ School of Electrical and Information Engineering \\ University of Sydney \\ NSW 2006, Australia \\ Email: ian.laird@sydney.edu.au
}

\author{
Dylan D.C. Lu \\ School of Electrical and Information Engineering \\ University of Sydney \\ NSW 2006, Australia \\ Email: dylan.lu@sydney.edu.au
}

\begin{abstract}
During operation thermoelectric generators (TEGs) are subject to the following thermal effects; Heat conduction according to Fourier's law, Joule heating, Peltier heating and Thomson heating. Many SPICE-based models exist for TEGs however in the vast majority of them the Thomson effect is neglected due to its relatively small size compared to the other effects, as well as the complexity that results from including the Thomson effect in the model. This paper seeks to present a model that governs the steady state performance of a TEG that includes the Thomson effect whilst limiting the complexity of the SPICE model.
\end{abstract}

\section{NOMENCLATURE}

$$
\begin{aligned}
A & =\text { Thermoelement cross-sectional area }\left(\mathrm{m}^{2}\right) \\
c & =\text { Specific heat }(\mathrm{J} / \mathrm{kg} \cdot \mathrm{K}) \\
I & =\text { Electrical current }(\mathrm{A}) \\
k & =\text { Thermal conductivity }(\mathrm{W} / \mathrm{m} \cdot \mathrm{K}) \\
L & =\text { Thermoelement length }(\mathrm{m}) \\
P_{e} & =\text { Output electrical power }(\mathrm{W}) \\
\dot{Q}_{C} & =\text { Cold junction heat rejection rate }(\mathrm{W}) \\
\dot{Q}_{H} & =\text { Hot junction heat absorption rate }(\mathrm{W}) \\
\dot{Q}_{J} & =\text { Joule heat generation rate }(\mathrm{W}) \\
\dot{Q}_{K} & =\text { Thermal conduction heat flow }(\mathrm{W}) \\
\dot{Q}_{P} & =\text { Peltier heat generation rate }(\mathrm{W}) \\
\dot{Q}_{S} & =\text { Generation rate of internal heat sources }(\mathrm{W}) \\
\dot{Q}_{T} & =\text { Thomson heat generation rate }(\mathrm{W}) \\
R & =\text { Thermoelement electrical resistance }(\Omega) \\
R_{L} & =\text { Electrical load }(\Omega) \\
t & =\text { Time }(\mathrm{s}) \\
T & =\text { Absolute temperature }(\mathrm{K}) \\
T_{C} & =\text { Cold junction temperature }(\mathrm{K}) \\
T_{H} & =\text { Hot junction temperature }(\mathrm{K}) \\
V & =\text { Thermocouple voltage }(\mathrm{V}) \\
x & =\text { Thermoelement axial position from the hot }
\end{aligned}
$$

This project was sponsored by an Australian Postgraduate Award (APA) and the Norman I. Price scholarship

Corresponding author contact: ian.laird@sydney.edu.au

$$
\begin{array}{rll} 
& \text { junction }(\mathrm{m}) \\
\alpha= & \text { Seebeck coefficient }(\mathrm{V} / \mathrm{K}) \\
\beta= & \text { Thomson coefficient }(\mathrm{V} / \mathrm{K}) \\
\rho= & \text { Electrical resistivity }(\Omega \cdot \mathrm{m}) \\
\rho_{d}= & \text { Mass density }\left(\mathrm{kg} / \mathrm{m}^{3}\right) \\
& \text { I. INTRODUCTION }
\end{array}
$$

\section{INTRODUCTION}

The search for sustainable and non-polluting electrical energy sources over the years has become a major pursuit for a great number of people involved in a wide range of fields. As environmental problems worsen and the demand for energy increases, energy sources that were once considered novelties are now essential parts of the research and development (R\&D) activities being undertaken to create a solution for the energy problem. One of the energy sources undergoing $R \& D$ is the thermoelectric generator (TEG).

A thermoelectric module (TEM) is a solid state device that converts a temperature gradient directly into electricity (by which it is known as a TEG) and also, electricity back into a temperature gradient (by which it is known as a thermoelectric cooler (TEC)). It consists of a large number of thermocouples that are connected electrically in series and thermally in parallel. The thermocouples are junctions of heavily doped semiconductors. When used as a TEG, heat is applied to one junction while it is removed from the other. This causes electrons in the n-type leg and holes in the p-type leg to drift away from the hot junction towards the cold one as shown in Fig. 1. The resulting charge separation produces a voltage across the thermocouple. This is known as the Seebeck voltage and its magnitude is proportional to the temperature difference of the hot and cold junctions [1]. If a load is connected across the thermocouple, a DC current flows.

A range of SPICE models have been developed to assist the design of power electronic converters that use TEGs as power sources. These models typically consist of two parts; one part modelling the electrical effects of a TEG and the other the thermal effects. TEG operation is governed by the following thermal effects; Thermal conduction, Joule heating, Peltier heating and cooling, and Thomson heating. Due to its relatively small impact, the Thomson effect is often neglected from SPICE models. Chavez [2] includes the Thomson effect 


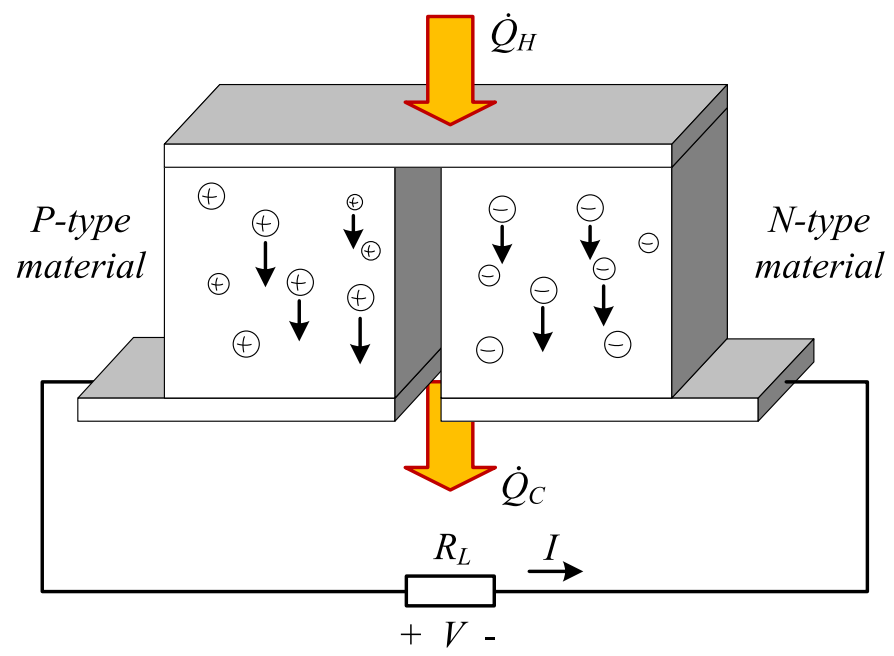

Fig. 1. Thermocouple operation in generator mode

TABLE I

THERMAL-ELECTRICAL ANALOGIES

\begin{tabular}{c|c}
\hline Thermal property & Electrical property \\
\hline Temperature difference $(\mathrm{K})$ & Voltage $(\mathrm{V})$ \\
Quantity of heat $(\mathrm{J})$ & Charge $(\mathrm{C})$ \\
Heat flow rate $(\mathrm{W})$ & Current $(\mathrm{A})$ \\
Thermal resistance $(\mathrm{K} / \mathrm{W})$ & Electrical resistance $(\Omega)$ \\
Thermal capacitance $(\mathrm{J} / \mathrm{K})$ & Electrical capacitance $(\mathrm{F})$ \\
\hline
\end{tabular}

in the governing thermoelectric steady state differential equation even though the solution is simplified by assuming a linear temperature gradient across the thermoelements. The resulting SPICE model utilises equivalent electrical components, as shown in Table I, but neglects the Thomson effect. Similar models are developed in [3]-[5] and again the Thomson effect is neglected due to its relatively small impact compared to the other effects. Rodriguez [6] creates a model utilising repeating circuit blocks to generate a model that factors in the change in material properties with temperature. The Thomson effect is included in the analysis, however the simplified form of $\dot{Q}_{T}=\beta I \Delta T$ is used rather than that of (4). Similarly, Chen [7] uses numerical modelling to create a far more comprehensive TEG model from repeating circuit blocks however the complexity of the model is dramatically increased and the Thomson coefficient is ultimately removed from the equations in favour of $\mathrm{d} \alpha$.

This paper intends to develop a model that demonstrates the steady state performance of a TEG which includes the Thomson effect whilst maintaining a relatively simple and easy-touse SPICE model. The paper is organised as follows. Section II outlines the thermoelectric effects and formulates and solves the one-dimensional heat equation of a thermoelement. Section III and IV show the SPICE models that develop out of the heat equation for the thermoelements and thermocouples respectively. Section $\mathrm{V}$ analyses the impact of the Thomson effect on the TEG operation according to the simulation results. Finally conclusions are drawn in section VI.

\section{THERMOELECTRIC EFFECTS AND THE HEAT EQUATION}

As mentioned above, the operation of a TEG is governed by thermal conduction, Joule heating, Peltier heating and cooling, and Thomson heating. Thermal conduction is the transfer of heat energy from particles at high temperature, and thus high energy, to ones at low temperature [8] according to (1). Joule heating is the heat generated by the flow of electric current through a conductor according to (2). Peltier heating/cooling for a TEG is the heat energy absorbed/emitted at the hot/cold junction of a thermocouple in order to generate an electron flow within the device. The Peltier effect is reversible in that driving an electric current through the device will cause one junction to heat up and the other to cool down. The rate at which heat is absorbed is given by (3). It should be noted that the polarity of the Seebeck coefficient is reversed at the cold junction resulting in the equation delivering a negative result thus indicating the rate at which heat is emitted. Thomson heating is the generation of heat as a result of an electric current flowing along a conductor that is also subject to a temperature gradient. Likewise, this effect is also reversible and the rate at which heat is generated is given by (4). Again the polarity of the current, temperature gradient and Thomson coefficient can be reversed meaning that if the result of (4) is positive then heat is emitted by the thermoelement, while a negative result means it is absorbed.

$$
\begin{aligned}
\dot{Q}_{K} & =-k A \frac{\mathrm{d} T}{\mathrm{~d} x} \\
\dot{Q}_{J} & =\int \frac{\rho I^{2}}{A} \mathrm{~d} x \\
\dot{Q}_{P} & =\alpha_{p n} T I \\
\dot{Q}_{T} & =-\int \beta I \frac{\mathrm{d} T}{\mathrm{~d} x} \mathrm{~d} x
\end{aligned}
$$

Note that only the Peltier effect is unaffected by the length of the thermoelement as it is a phenomenon observed at the junctions as emphasised by the $p n$ subscript. The generic equation that models the conduction of heat through a onedimensional rod is comprehensively derived in [9] and shown graphically in Fig. 2, and is repeated here with some modification as the equation for heat conduction for a thermoelement.

$$
c \rho_{d} A \frac{\partial T}{\partial t}=A \frac{\partial}{\partial x}\left(k \frac{\partial T}{\partial x}\right)+\frac{\partial}{\partial x} \dot{Q}_{S}
$$

Joule and Thomson heating are the internal heat sources of a thermoelement. Therefore using $\dot{Q}_{S}=\dot{Q}_{J}+\dot{Q}_{T}$ and the fact that for steady state operation $\frac{\partial T}{\partial t}=0,(5)$ becomes the following ordinary differential equation (ODE)

$$
k A \frac{\mathrm{d}^{2} T}{\mathrm{~d} x^{2}}-\beta I \frac{\mathrm{d} T}{\mathrm{~d} x}=-\frac{\rho I^{2}}{A}
$$

The positive direction of $x$ and $I$ are defined in Fig. 2. This equation is the same as that specified in [2]. Using the boundary conditions $T(0)=T_{H}$ and $T(L)=T_{C}$, (6) is solved to give 


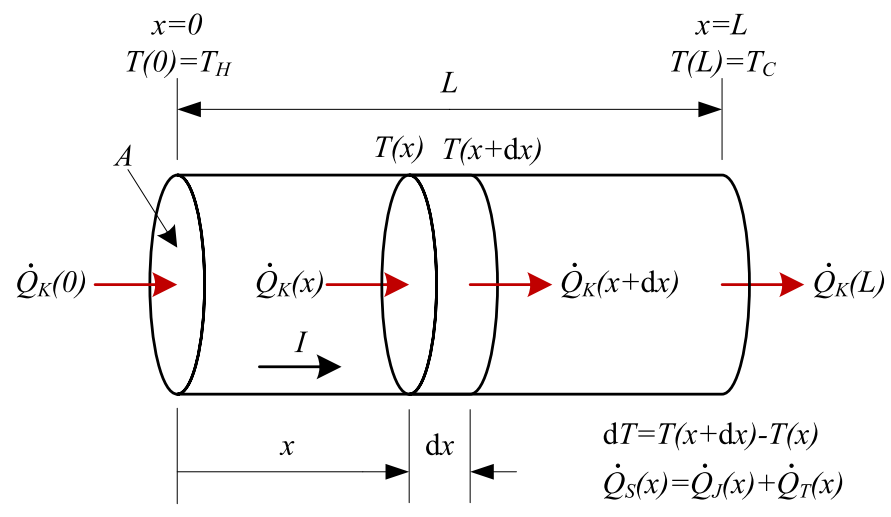

Fig. 2. Heat flow within a thermoelement subject to thermoelectric effects

$$
T(x)=T_{H}+\frac{\rho I}{\beta A} x-\left(T_{H}-T_{C}+\frac{\rho I}{\beta A} L\right) \frac{1-e^{\frac{\beta I}{k A} x}}{1-e^{\frac{\beta I}{k A} L}}
$$

This is same as [10] except for the opposite polarity sign of the terms containing the Thomson coefficient. This is because in [10] the ordinary differential equation that was solved used a positive sign for the Thomson heating effect whereas [2] and this paper use a negative one. This conflict can be cleared up by expressing the Thomson effect as a voltage given by $V=\beta T$, even though the effect in of itself is not a voltage [11]. This states that a thermoelement can be considered as having a voltage varying along its length and that a current passing through this will result in energy being absorbed or emitted depending on whether it is travelling from a low to high or high to low potential respectively. The positive/negative Thomson effect is when a high temperature corresponds to a high/low potential and thus requires a positive/negative Thomson coefficient respectively. Therefore in order to represent the Thomson heating term in (6) as an internal heat source rather than a sink, given our defined positive directions of $x$ and $I$, the term requires a negative sign.

From (7) it can be seen that the temperature profile is not linear (as is often assumed) due to the effect of the internal heat sources. Differentiating (7) and multiplying by $-k A$ gives the rate of heat conduction at position $x$

$$
\dot{Q}_{K}(x)=-\frac{\rho k I}{\beta}-\beta I\left(T_{H}-T_{C}+\frac{\rho I}{\beta A} L\right) \frac{e^{\frac{\beta I}{k A} x}}{1-e^{\frac{\beta I}{k A} L}}
$$

Similar to before, (8) shows that the amount of heat flowing through the thermoelement due to conduction, varies with position due to the internal heat sources. Many models assume a linear temperature profile which results in a constant heat flow due to conduction.

\section{SPICE MODEL OF THERMOELEMENTS}

SPICE is used, among other things, to model the time domain response of a circuit. It operates by generating the $\mathrm{KCL}$ and KVL equations and solving them using matrix

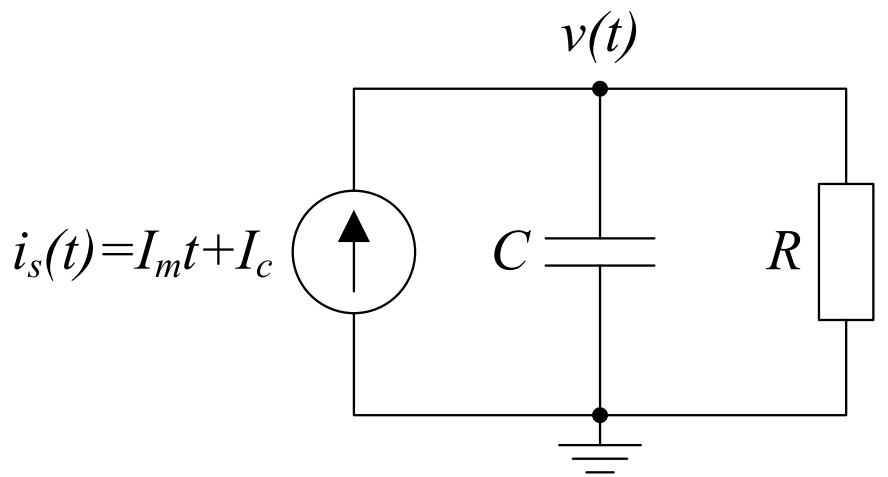

Fig. 3. Electrical circuit model of a thermoelement

mathematics presenting the results as voltages and currents as functions of time. RLC circuits give rise to 2nd-order ODEs similar to that of (6) except that they are functions of $t$ rather than $x$. Therefore with the correct model, the time domain response produced by SPICE can be used to model the steady state temperature profile within a thermoelement where the time scale of the output now represents the axial position within the thermoelement. In order to develop a model based on an RLC circuit, the governing equation needs to be of the form $a \frac{\mathrm{d}^{2} y}{\mathrm{~d} t^{2}}+b \frac{\mathrm{d} y}{\mathrm{~d} t}+c t$ where $y$ represents voltage or current and $a, b, c$ are non-zero. Unfortunately for (6) $c=0$, however if (6) is integrated with respect to $x$ the result is

$$
k A \frac{\mathrm{d} T}{\mathrm{~d} x}-\beta I T=-\frac{\rho I^{2}}{A} x+\dot{Q}_{U}
$$

where $\dot{Q}_{U}$ is a constant that is considered to be an unknown internal heat source. The form of (9) suggests that the choice of model should be a first-order circuit subject to a source of the form $y(t)=Y_{m} t+Y_{c}$. Keeping with the accepted conventions shown in Table I a parallel RC circuit with a current source is chosen for the model as shown by Fig. 3. Performing nodal analysis the governing equation is derived to be

$$
C \frac{\mathrm{d} v}{\mathrm{~d} t}+\frac{1}{R} v=I_{m} t+I_{c}
$$

Comparing this with (9) and its boundary conditions we get the following relationships

$$
\begin{aligned}
C & =k A \\
R & =-\frac{1}{\beta I} \\
I_{m} & =-\frac{\rho I^{2}}{A} \\
I_{c} & =\dot{Q}_{U}
\end{aligned}
$$

Solving (9) gives

$$
T(x)=\frac{\rho I}{\beta A} x-\frac{\dot{Q}_{U}}{\beta I}+\frac{\rho k}{\beta^{2}}+\left(T_{H}+\frac{\dot{Q}_{U}}{\beta I}-\frac{\rho k}{\beta^{2}}\right) e^{\frac{\beta I}{k A} x}
$$



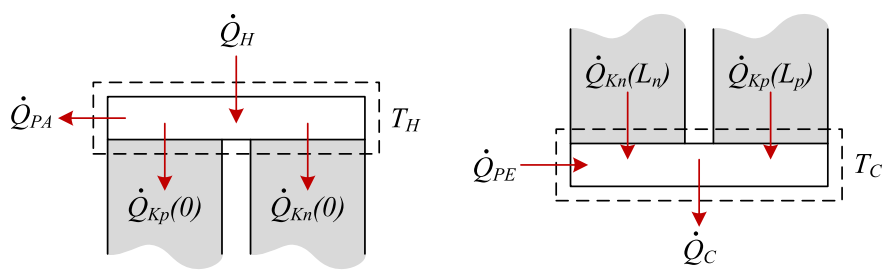

Fig. 4. Hot and cold junction thermodynamic systems

Comparing the coefficients of $e^{\frac{\beta I}{k A} x}$ from (7) and (11) produces the following equation

$$
T_{H}+\frac{\dot{Q}_{U}}{\beta I}-\frac{\rho k}{\beta^{2}}=\frac{T_{H}-T_{C}+\frac{\rho I}{\beta A} L}{1-e^{\frac{\beta I}{k A} L}}
$$

Solving (12) for $\dot{Q}_{U}$ gives

$$
\dot{Q}_{U}=\frac{\rho k I}{\beta}-\beta I T_{H}+\frac{\frac{\rho L}{A} I^{2}+\beta I\left(T_{H}-T_{C}\right)}{1-e^{\frac{\beta I}{k A} L}}
$$

The initial and final conditions of the capacitor are set to $v(0)=V_{H}$ and $v(T)=V_{L}$ to mimic the boundary conditions $T(0)=T_{H}$ and $T(L)=T_{C}$ respectively, and thus complete the model. It should be noted that $\mathrm{n}$ - and $\mathrm{p}$ type thermoelements possess different values for $k, \rho$ and $\beta$, and depending on the construction $L$ and $A$ might be different as well. As a result $n$ and $p$ subscripts are used to distinguish between equations relating to $n$ - and p-type materials respectively. Finally as shown in Fig. 1, the direction of $I$ is reversed for an n-type thermoelement and must be accounted for when evaluating the above equations.

\section{SPICE MODEL FOR THERMOCOUPLES}

In order to create a model that is useful for designing power electronic converters, a model of the heat flows at the thermocouple junctions is required. To achieve this the heat conduction rates at the endpoints of the thermoelements and the Peltier effects are required. To analyse the junctions, thermodynamic system boundaries are drawn around them and the heat and work flows across the boundaries are identified as shown in Fig. 4.

It should be noted that all the energy flows are heat with the exception of $\dot{Q}_{P A}$ and $\dot{Q}_{P E}$ which are actually the rate at which energy is transferred to or from the electron flow as it passes through the junction. In essence $\dot{Q}_{P A}$ and $\dot{Q}_{P E}$ are the proportions of $\dot{Q}_{H}$ and $\dot{Q}_{C}$ respectively, that are absorbed or emitted by the electron flow hence the $P A$ and $P E$ notation used. It is important to note that even though the electrons are gaining/losing energy, since they flow in and out of the system at a steady rate, they do not cause a change to the internal energy of the system. Therefore the assumption that the system is in steady state is true. Performing an energy balance on the hot and cold junctions produces the following equations

$$
\dot{Q}_{H}=\dot{Q}_{K p}(0)+\dot{Q}_{K n}(0)+\dot{Q}_{P A}
$$

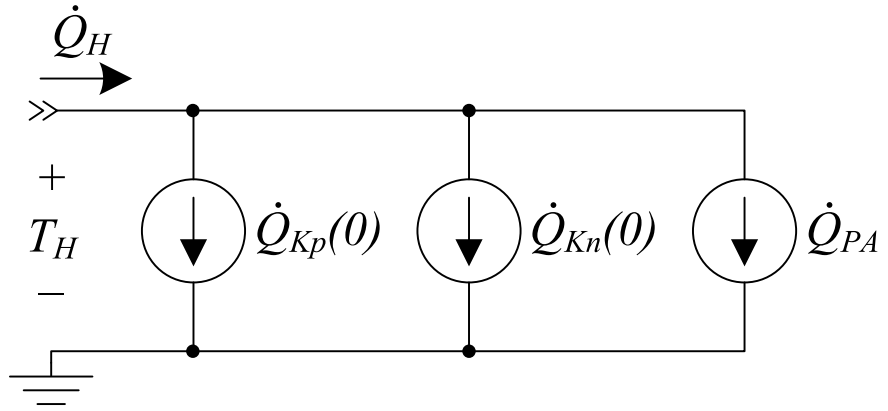

Fig. 5. Electrical circuit model of a thermocouple hot junction

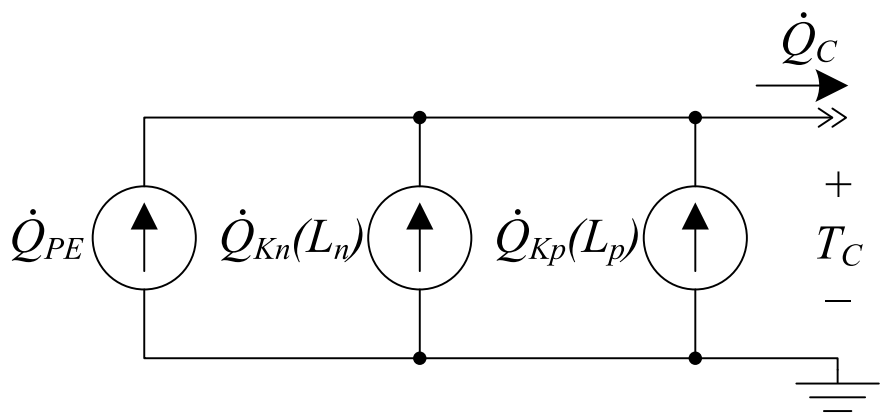

Fig. 6. Electrical circuit model of a thermocouple cold junction

$$
\dot{Q}_{C}=\dot{Q}_{K p}\left(L_{p}\right)+\dot{Q}_{K n}\left(L_{n}\right)+\dot{Q}_{P E}
$$

The models for the hot and cold junctions are derived from (14) and (15) and are shown in Fig. 5 and Fig. 6 respectively. To complete the thermocouple model, an electrical sub-model is required. This can be obtained by substituting (3) and (8) into (14) and (15) to give

$$
\begin{aligned}
\dot{Q}_{H}= & \left(\frac{\rho_{n} k_{n}}{\beta_{n}}-\frac{\rho_{p} k_{p}}{\beta_{p}}\right) I+\frac{\beta_{n} I\left(T_{H}-T_{C}\right)-\frac{\rho_{n} L_{n}}{A_{n}} I^{2}}{1-e^{-\frac{\beta_{n} L_{n}}{k_{n} A_{n}} I}} \\
& -\frac{\beta_{p} I\left(T_{H}-T_{C}\right)+\frac{\rho_{p} L_{p}}{A_{p}} I^{2}}{1-e^{\frac{\beta_{p} L_{p}}{k_{p} A_{p}} I}}+\alpha_{p n} T_{H} I \\
\dot{Q}_{C}= & \left(\frac{\rho_{n} k_{n}}{\beta_{n}}-\frac{\rho_{p} k_{p}}{\beta_{p}}\right) I-\frac{\beta_{n} I\left(T_{H}-T_{C}\right)-\frac{\rho_{n} L_{n}}{A_{n}} I^{2}}{1-e^{\frac{\beta_{n} L_{n}}{k_{n} A_{n}} I}} \\
& +\frac{\beta_{p} I\left(T_{H}-T_{C}\right)+\frac{\rho_{p} L_{p}}{A_{p}} I^{2}}{1-e^{-\frac{\beta_{p} L_{p}}{k_{p} A_{p}} I}}+\alpha_{p n} T_{C} I
\end{aligned}
$$

From (16) and (17) the output electrical power and voltage of the thermocouple are determined to be

$$
\begin{aligned}
P_{e} & =\dot{Q}_{H}-\dot{Q}_{C} \\
& =\left(\alpha_{p n}+\beta_{n}-\beta_{p}\right)\left(T_{H}-T_{C}\right) I-\left(\frac{\rho_{p} L_{p}}{A_{p}}+\frac{\rho_{n} L_{n}}{A_{n}}\right) I^{2}
\end{aligned}
$$




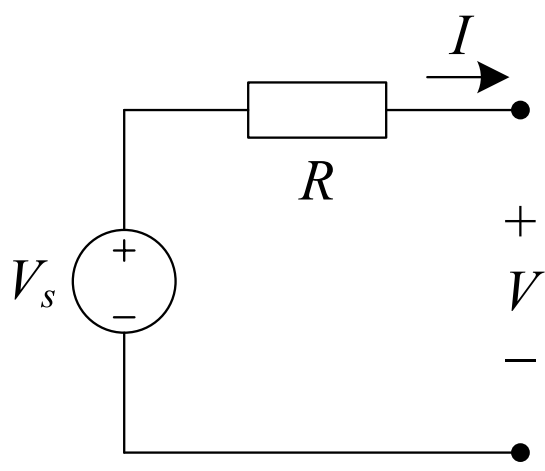

Fig. 7. Sub-model of thermocouple electrical effects

$$
\begin{aligned}
V & =\frac{P_{e}}{I} \\
& =\left(\alpha_{p n}+\beta_{n}-\beta_{p}\right)\left(T_{H}-T_{C}\right)-\left(\frac{\rho_{p} L_{p}}{A_{p}}+\frac{\rho_{n} L_{n}}{A_{n}}\right) I
\end{aligned}
$$

The thermocouple electrical sub-model is derived from (19) to be a Thévenin equivalent circuit with the parameters $V_{s}=$ $\left(\alpha_{p n}+\beta_{n}-\beta_{p}\right)\left(T_{H}-T_{C}\right)$ and $R=\frac{\rho_{p} L_{p}}{A_{p}}+\frac{\rho_{n} L_{n}}{A_{n}}$ as shown in Fig. 7. (18) and (19) show that altering the Thomson effect will not change the shape of the P-I and V-I characteristics, that is they will remain parabolic and linear respectively with some changes in magnitude depending on the values of $\beta_{n}$ and $\beta_{p}$. For a typical TEG SPICE model that disregards the Thomson effect as shown in [2], [5], $V_{s}$ is given by $V_{s}=$ $\alpha_{p n}\left(T_{H}-T_{C}\right)$ which matches the above model evaluated for $\beta_{n}=\beta_{p}=0$. Therefore the electrical characteristics of the above thermocouple model closely matchs that of previously defined models.

\section{Simulation ANALYSis AND EXPERIMENTAL COMPARISON}

Applying non-dimensional analysis to (7) and (8) the following equations are obtained

$$
\begin{gathered}
T^{*}=1+\frac{x^{*}}{\dot{Q}_{T / J}}-\left(1+\frac{1}{\dot{Q}_{T / J}}\right) \frac{1-e^{\dot{Q}_{T / K} x^{*}}}{1-e^{\dot{Q}_{T / K}}} \\
\dot{Q}^{*}=-\frac{1}{\dot{Q}_{T / J}}-\dot{Q}_{T / K}\left(1+\frac{1}{\dot{Q}_{T / J}}\right) \frac{e^{\dot{Q}_{T / K} x^{*}}}{1-e^{\dot{Q}_{T / K}}} \\
\text { where }\left\{\begin{aligned}
x^{*} & =\frac{x}{L} \\
T^{*} & =\frac{T(x)-T_{C}}{T_{H}-T_{C}} \\
\dot{Q}^{*} & =\frac{L \dot{Q}_{K}(x)}{k A\left(T_{H}-T_{C}\right)} \\
\dot{Q}_{T / K} & =\frac{\beta I L}{k A} \\
\dot{Q}_{T / J} & =\frac{\beta A\left(T_{H}-T_{C}\right)}{\rho I L}
\end{aligned}\right.
\end{gathered}
$$

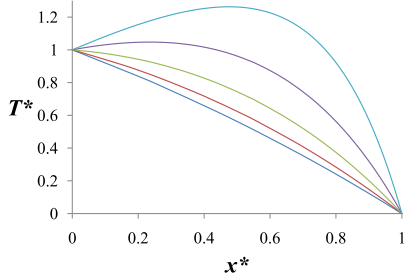

(a)

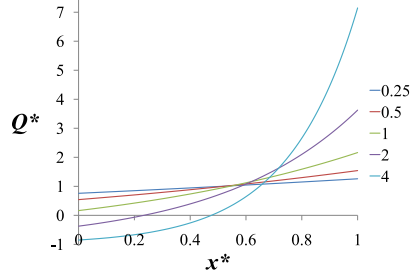

(b)
Fig. 8. Non-dimensional temperature and heat flow profile of a thermoelement with $\dot{Q}_{T / J}=1$ and varying values of $\dot{Q}_{T / K}$

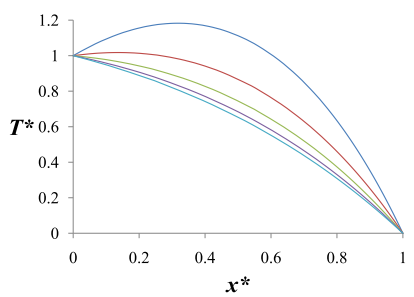

(a)

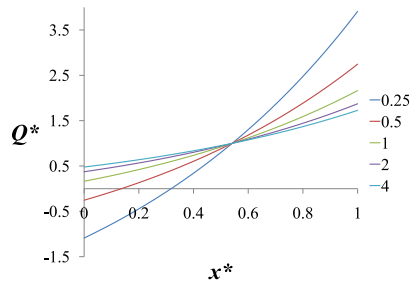

(b)
Fig. 9. Non-dimensional temperature and heat flow profile of a thermoelement with $\dot{Q}_{T / K}=1$ and varying values of $\dot{Q}_{T / J}$

$x^{*}, T^{*}$ and $\dot{Q}^{*}$ are the non-dimensional position, temperature and heat conduction of the thermoelement respectively. $\dot{Q}_{T / K}$ and $\dot{Q}_{T / J}$ represent the ratios of the Thomson heating to heat conduction and Thomson to Joule heating respectively, that result from a thermoelement subject to a linear temperature profile. Fig. 8 shows the effect of varying the Thomson effect with respect to the heat conduction rate when the Thomson and Joule effects are equal. As can be seen in Fig. 8a, increasing the Thomson effect results in an increasingly nonlinear temperature profile. Also as shown in Fig. 8b, if the Thomson effect is greater than the heat conduction rate, the temperature at various positions along the thermoelement will be greater than $T_{H}$ and the heat will flow back into the hot junction from positions before the maximum temperature. Fig. 9 shows the effect on the temperature profile of varying the Thomson effect with respect to the Joule effect when Thomson and conduction effects are equal. Unlike before increasing the Thomson effect results in an increasingly linear temperature profile as shown in Fig. 9a. Again the temperature can be greater than $T_{H}$ causing the heat to flow back to the hot junction however this time it is due to the Joule effect being larger than the Thomson effect as shown in Fig. 9b. One other observation from Fig. $9 \mathrm{~b}$ is that the point at which the actual heat conduction rate is equal to that of the "linear" one, is always the same for a constant value of $\dot{Q}_{T / K}$. Overall the temperature profile will tend to be non-linear if the Thomson effect is large with comparison to the heat conduction rate, yet small compared to the Joule heat effect.

The main advantage of using a thermoelement model in combination with one for a thermocouple is that it allows for a more accurate selection of the TEG steady state parameters. 


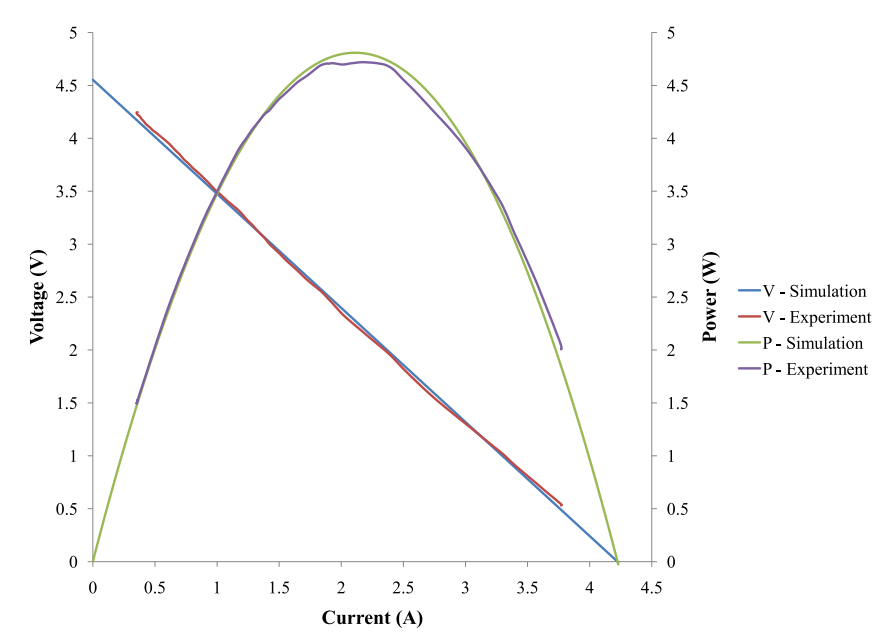

Fig. 10. Simulated and experimentally obtained V-I and P-I characteristics of a TEP1-12656-0.6 subject to $T_{H}=495$ and $T_{C}=320$

Since TEG parameters vary with temperature they are typically selected based on their values that correspond to the average temperature of the thermoelement. The average temperature is often approximated as $\bar{T}=\frac{T_{H}+T_{C}}{2}$. However this is only correct if the temperature profile is linear. Using (7) the average temperature can be derived as:

$$
\begin{aligned}
\bar{T} & =\frac{1}{L} \int_{0}^{L} T(x) \mathrm{d} x \\
& =T_{H}+\frac{\rho I L}{2 \beta A}-\frac{k A\left(T_{H}-T_{C}\right)}{\beta I L}-\frac{\rho k}{\beta^{2}}-\frac{T_{H}-T_{C}+\frac{\rho I L}{\beta A}}{1-e^{\frac{\beta I L}{k A}}}
\end{aligned}
$$

Hence by using (22), a more accurate average temperature can be used for selecting the parameters.

Finally, a simple experimental verification of the thermocouple electrical sub-model was performed by measuring the V-I and P-I characteristics of a TEP1-12656-0.6 thermoelectric module for hot side and cold side temperatures of $495 \mathrm{~K}$ and $320 \mathrm{~K}$ respectively. The simulation was defined by the two lumped parameters $V_{s}$ and $R$ shown above where $V_{s}=4.5538$ and $R=1.0779$. The simulation and the experimental data of the V-I and P-I characteristics are compared in Fig. 10. As can be seen, the linear V-I and the parabolic P-I characteristics of the simulation closely match that of the experimental results.

\section{CONCLUSION}

This paper has presented SPICE based models for both a thermoelement and a thermocouple where the Thomson effect is included. The temperature profile and the heat conduction flows were derived using the general heat equation. Analysis of the thermoelement model revealed that the linearity of the temperature profile is dependent on the relative sizes of the heat conduction rate, Joule effect and Thomson effect. This gave rise to a revised way of calculating the average temperature of a thermoelement which takes into account the non-linearity of the element's temperature profile. Lastly the thermocouple electrical sub-model was experimentally verified.

\section{REFERENCES}

[1] S. B. Riffat and X. Ma, "Thermoelectrics: a review of present and potential applications," Applied Thermal Engineering, vol. 23, no. 8, pp. 913 - 935, 2003. [Online]. Available: http://www.sciencedirect.com/science/ article/B6V1Y-485P6M3-1/2/10905dcee9791673dcf7fd35280522f9

[2] J. Chavez, J. Ortega, J. Salazar, A. Turo, and M. Garcia, "Spice model of thermoelectric elements including thermal effects," in Instrumentation and Measurement Technology Conference, 2000. IMTC 2000. Proceedings of the 17th IEEE, vol. 2, 2000, pp. $1019-1023$.

[3] J. G. Vián, D. Astrain, and M. Domnguez, "Numerical modelling and a design of a thermoelectric dehumidifier," Applied Thermal Engineering, vol. 22, no. 4, pp. 407 - 422, 2002. [Online]. Available: http://www.sciencedirect.com/science/article/ B6V1Y-44HYFMJ-1/2/290688d9fa8a8acad6ce71731087b73f

[4] D. Astrain, J. Vián, and J. Albizua, "Computational model for refrigerators based on peltier effect application," Applied Thermal Engineering, vol. 25, no. 17-18, pp. 3149 - 3162 , 2005. [Online]. Available: http://www.sciencedirect.com/science/article/ B6V1Y-4G9Y5JJ-6/2/10c9bad83c97876dc6d060f9c08f6941

[5] S. Lineykin and S. Ben-Yaakov, "Modeling and analysis of thermoelectric modules," Industry Applications, IEEE Transactions on, vol. 43, no. 2, pp. 505 -512, Mar.-Apr. 2007.

[6] A. Rodriguez, J. Vián, D. Astrain, and A. Martinez, "Computational model and test bench for thermoelectric power generation, for thermoelectric parameters dependent on the temperature," in Thermoelectrics, 2006. ICT '06. 25th International Conference on, Aug. 2006, pp. 300 -304 .

[7] M. Chen, L. Rosendahl, T. Condra, and J. Pedersen, "Numerical modeling of thermoelectric generators with varing material properties in a circuit simulator," Energy Conversion, IEEE Transactions on, vol. 24, no. 1 , pp. $112-124$, Mar. 2009.

[8] Y. A. Çengel and R. H. Turner, Fundamentals of Thermal-Fluid Sciences, 2nd ed. McGraw-Hill, 2005.

[9] R. Haberman, Applied Partial Differential Equations with Fourier Series and Boundary Value Problems, 4th ed. Pearson Prentice Hall, 2004.

[10] J. Sunderland and N. T. Burak, "The influence of the thomson effect on the performance of a thermoelectric power generator," Solid-State Electronics, vol. 7, no. 6, pp. 465 - 471, 1964. [Online]. Available: http://www.sciencedirect.com/science/article/ B6TY5-46TYN6V-44/2/da8e054a9e9c568faa1ba514bc4a0db0

[11] D. M. Rowe, Ed., CRC Handbook of Thermoelectrics. CRC Press, 1994, ch. 2, pp. $7-17$. 